\title{
Midgut volvulus as a complication of intestinal malrotation in a term pregnancy
}

\author{
Sung Mi Hwang ${ }^{1}$, Yeon Sik Na ${ }^{2}$, Young $\mathrm{Cho}^{2}$, Dong Guen You', and Jae Jun Lee ${ }^{1}$ \\ Departments of ${ }^{1}$ Anesthesiology and Pain Medicine, ${ }^{2}$ Obstetrics and Gynecology, Hallym University College of Medicine, \\ Chuncheon, Korea
}

Intestinal malrotation is an uncommon cause of abdominal pain and normally presents during infancy. Approximately $90 \%$ of patients with malrotation are diagnosed within the first year of life, $80 \%$ of whom are diagnosed within the first month of life [1]. Intestinal malrotation complicated by midgut volvulus, a well recognized disease entity in infants and children, is rare in adults [2]. We report a pregnant woman with rare small and large bowel infarctions due to intestinal malrotation complicated by midgut volvulus whose signs and symptoms were misunderstood as labor.

A 22-year-old primigravida at 38 weeks and 2 days gestation who had been followed during pregnancy at a private obstetric clinic visited our emergency room with the chief complaint of labor pain that had developed $1 \mathrm{~h}$ and $30 \mathrm{~min}$ previously. On admission, her vital signs were blood pressure 130/90 mmHg and heart rate 83 beats/min, however, the patient suddenly developed hypotension $(80 / 50 \mathrm{mmHg})$ and tachycardia (134 beats/min) $7 \mathrm{~h}$ later, and the fetal heart rate trace decreased from 120-130 to $90-100$ beats/min.

An emergency Cesarean section was performed and a male newborn $(2,870 \mathrm{~g})$ was delivered with an Apgar score of 1 at $1 \mathrm{~min}$. Emergency resuscitation was initiated, at the same time, a $3.0 \mathrm{~mm}$ uncuffed endotracheal tube was intubated by the anesthesiologist. The $5 \mathrm{~min}$ Apgar score was 4, and he was transferred to the neonatal intensive care unit by a pediatric physician. The obstetric physician found an ischemic change in the small intestine above the uterus of the mother (Fig. 1A). A general surgeon was called, and upon entering the abdominal
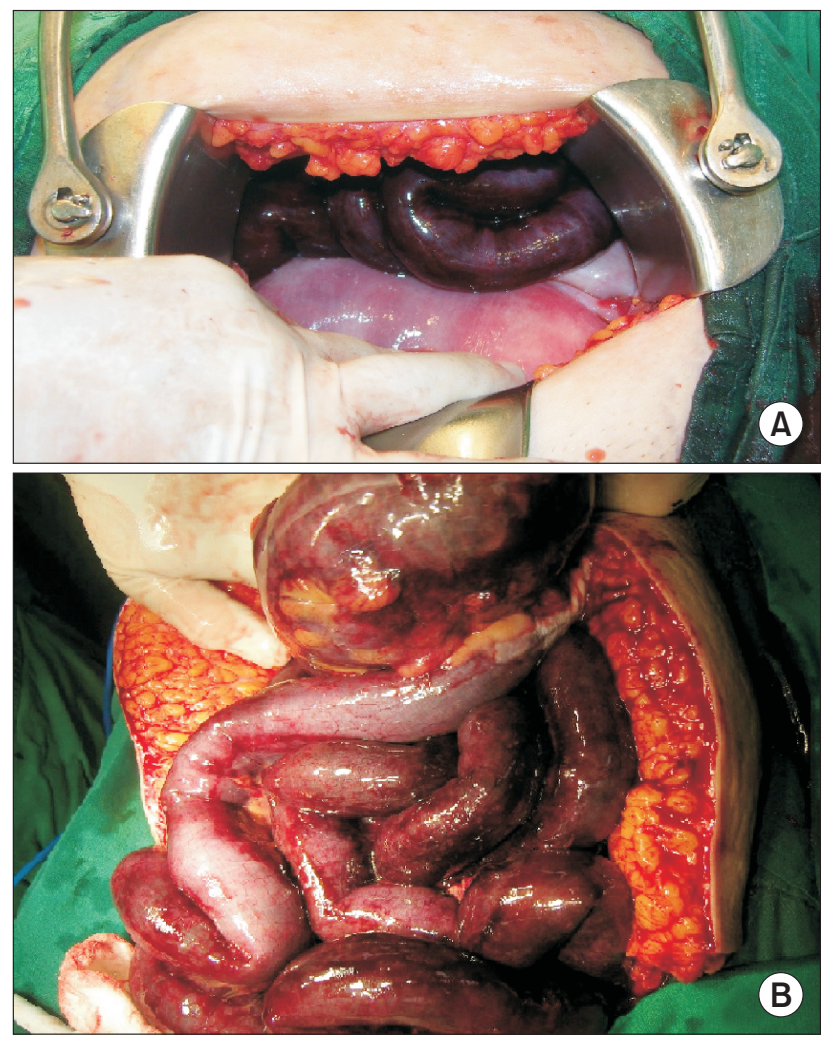

Fig. 1. (A) Small intestine with ischemic change above the uterus. (B) The ischemic intestine due to volvulus from the proximal transverse colon, with the ischemic portion extending toward to Treitz's ligament of the small intestine. The ischemic ascending colon was located in the middle of the peritoneal cavity.

Corresponding author: Jae Jun Lee, M.D., Department of Anesthesiology and Pain Medicine, Hallym University College of Medicine, 77, Sakjuro, Chuncheon 200-704, Korea. Tel: 82-33-240-5594, Fax: 82-33-251-0941, E-mail: iloveu59@hallym.or.kr

(c) This is an open-access article distributed under the terms of the Creative Commons Attribution Non-Commercial License (http:// creativecommons.org/licenses/by-nc/3.0/), which permits unrestricted non-commercial use, distribution, and reproduction in any medium, provided the original work is properly cited. 
cavity, the areas of ischemic change were observed from Treitz's ligament of the small intestine to the proximal transverse colon, but no perforation was detected. A congenital intestinal malrotation and mobile colon were observed, and the ischemic ascending colon was located in the middle of the peritoneal cavity (Fig. 1B). The ischemic portion of the ascending colon was completely gangrenous; thus, a right hemicolectomy was performed. Unfortunately, the mother died 2 days after surgery. However, the baby had no major medical problems and was discharged 2 weeks after birth.

In the present case, the mother had a congenital intestinal malrotation of which she was unaware, and the malrotation was complicated by midgut volvulus at full-term pregnancy; thus, the abdominal pain was misunderstood as labor pain. Furthermore, the physical findings associated with a midgut volvulus during pregnancy can be confusing and are not always those of a classic bowel obstruction [3]. Bowel sounds may be normal, and distention may be absent. Furthermore, she unfortunately had no nausea, vomiting, or constipation. The only abnormal finding was an elevated WBC count before she showed unstable vital signs.

The duodenum and cecum incompletely rotate and become close in proximity in cases of intestinal malrotation. This malpositioning results in a short stalk of mesentery that easily twists upon itself, resulting in compression of the superior mesenteric artery. This vascular compression results in ischemia of the intestine and necrosis of the intestinal wall in 1-2 $\mathrm{h}$ if left untreated [4], and the necrosis can compromise fetal health. Thus, a high level of diagnostic suspicion is needed for an early diagnosis in such a case.

Anesthesiologists should be aware of these conditions and similar cases to rapidly and definitively control vital signs, replace volume, and correct an electrolyte imbalance during anesthesia and to prepare for resuscitation of the neonate if needed. Knowledge of the management of this condition by anesthesiologists may decrease morbidity and mortality of the mother and neonate.

\section{References}

1. von Flüe M, Herzog U, Ackermann C, Tondelli P, Harder F. Acute and chronic presentation of intestinal nonrotation in adults. Dis Colon Rectum 1994; 37: 192-8.

2. Cathcart RS 3rd, Williamson B, Gregorie HB Jr, Glason PF. Surgical treatment of midgut nonrotation in the adult patient. Surg Gynecol Obstet 1981; 152: 207-10.

3. Stavorovsky M, Ilellin A, David M, Weintraub S. Midgut volvulus with secondary thrombosis of superior mesenteric vessels in a pregnant woman. Surgery 1977; 81: 353-6.

4. Torres AM, Zeigler MM. Malrotation of the intestine. World J Surg 1993; 17: 326-31. 